\title{
Senescence Markers: One is not Good Enough, We Need More!
}

\section{Gadhikar MA ${ }^{1}$ and Singh $\mathrm{PK}^{2 *}$}

${ }^{1}$ Department of Head and Neck Surgery, University of Texas, MD Anderson Cancer Center, USA

${ }^{2}$ Department of Experimental Radiation Oncology, University of Texas, MD Anderson Cancer Center, USA

*Corresponding author: Pankaj Kumar Singh, University of Texas, Anderson Cancer Center, US, Tel: 001 713-792-2121; E-mail: PKSingh@mdanderson.org

\section{Review Article}

Volume 1 Issue 2

Received Date: September 27, 2016

Published Date: October 20, 2016
DOI: $10.23880 /$ oajmb-16000108

\section{Abstract}

Senescence is considered as the response outcome generated in cells to halt the development and progression of tumor. The detection of senescent cells is problematic as there exists no exclusive marker of senescence that can be applied universally. In this review, we briefly describe the markers that are commonly used to identify senescent cells and also point out the limitations of their individual usage. We propose that rather than single marker, a combination of markers should be employed for faithful senescence detection and that increasing the available pool of senescence markers may facilitate senescence detection across different settings.

Keywords: Senescence Markers; Senescent Cells; Mitogenic Stimulus; $\beta$-galactosidase Activity; P16ink4a Expression

\section{Introduction}

Senescence is described as a terminally nonproliferative state of cells, characterized by a permanent exit from the cell cycle and inability to mount response to mitogenic stimulus $[1,2]$. Senescence can be induced by a range of cell intrinsic and extrinsic stimuli and is believed to pose a defensive barrier against tumor development and progression $[2,3]$. One of the nagging challenges in the field is that there exists no unique marker to identify senescent cells. Although a number of senescence markers have been proposed, not all senescent cells will exhibit all of these markers. The presence of senescence markers are chiefly dependent on the tissue of origin as well as the genetic makeup of cells [3,4]. Here we attempt to briefly summarize the markers that are routinely employed to recognize senescent state and point out the limitation of their individual usage for senescence detection.

\section{$\beta$-galactosidase Activity}

Senescence associated $\beta$-gal (SA- $\beta$-gal) activity SA is most widely used senescence marker primarily because of the ease of assay set up and its effortless detection in tissues $[1,5,6]$. $\beta$-galactosidase is an endogenous enzyme found in the lysosomal environment ( $\mathrm{pH} 4-4.5)$ with a maximal activity. For the purpose of senescence detection, 


\section{Open Access Journal of Microbiology \& Biotechnology}

the SA- $\beta$-gal activity is measured in situ at a suboptimal pH 6.0 with substrate X-Gal. A positive SA- $\beta$-gal activity denotes higher expression level of the enzyme $[7,8]$. Intriguingly, SA- $\beta$-gal activity is also found elevated under normal conditions in autophagic cells exhibiting surge in lysosomal activity and in matured osteoclasts and macrophages [9-12]. Besides this, cultured quiescent/differentiated cells also display increases in SA$\beta$-gal activity when they are maintained for extended periods [6]. Therefore, the suitability of SA- $\beta$-gal activity detection as a unique senescence marker is clearly questionable.

\section{p16INK4A Expression}

Another marker that is commonly employed for senescence detection is p16 expression. Normal healthy tissues do not have any detectable expression levels of $\mathrm{p} 16$, however, the levels rise markedly in response to certain kinds of stresses or changes in normal tissue configuration commonly found during tumorigenesis and ageing. Although these factors make p16 seemingly advantageous for senescence identification, there are limitations to its utility as senescence marker. p16 expression is not manifested in all forms of senescence. Instead, other tumor suppressor genes are found over expressed in culture models of senescence $[13,14]$. Moreover, there are instances where p16 is found abundantly expressed yet cell do not exhibit nonproliferative phenotype $[15,16]$. A prototypic example is the high level expression of p16 found in HPV cancer caused by the disruption of negative feedback loop from inactive $\mathrm{Rb}$ [15]. Thus, the presence of senescence gene is not a definitive indicator of senescence as the senescence inducing effects of these genes can be nullified by mutations in the downstream effector molecules.

\section{p21CIP1 Expression}

While the expression of p16INK4A is detected in a broad range of senescence settings, another important cyclin dependent kinase inhibitor that is found to accumulate rather commonly in senescent cells is p21CIP1 [17]. Induction of p21CIP1 has been found in replicative senescence. Moreover, anti-cancer drug treatments are also known to induce senescence in cancer cells through stimulation of p21expression [18]. For example, cisplatin treatment of wild type p53 head and neck cancer cells lead to senescence phenotype that was dependent on increased p21expression [2,19]. Similarly, p21 dependent induction of senescence phenotype was observed in range of cancer cells after treatment with NEDD8 activating enzyme inhibitor [20]. Although these results suggest that $\mathrm{p} 21$ expression may be an attractive surrogate for therapy induced senescence detection, but contrary findings in other cancer models precludes its general application for senescence detection. For instance, fibrosarcoma and colon cancer cells devoid of p21 still exhibited senescent features in response to drug treatment or ionizing radiation [18]. Another aspect that deters wide application of p21 expression as senescence marker comes from the observation that p21 over expression can also induce other form of cellular death such as apoptosis [17]. Because of these limitations, p21 expression alone does not appear to be a reliable marker for senescence.

\section{DNA Damage Foci}

DNA damage is closely linked to senescence as the chief senescence inducing pathways are spurred by the action of DNA damage response (DDR) signaling cascade. Activated DDR signaling is widely detected by monitoring the expression level of phosphorylated H2AX on serine residue 139 [21,22]. This phosphorylated histone variant, also known as Gamma H2AX, can form punctate pattern in the nucleus which denotes DSBs and/or uncapped or eroded telomeres or sometimes can be evident as a pan nuclear stain (for example, under conditions of oxidative or replication stress) [23]. DNA damage foci are prominently observed at the telomere junction in cells undergoing replicative senescence $[13,24,25]$. Moreover, expression of DNA damage foci is also seen in nontelomere regions of cells undergoing other forms of senescence [26,27]. Inspite of this apparent association between DNA damage foci and senescence induction, DNA damage foci cannot employed as a distinguishing marker for senescence. This is because a number of cancer cells exhibiting persistent activation DDR pathway are seen to proliferate normally in culture [28]. Similarly, wide range of genotoxic insults in cells also trigger DNA damage foci without eliciting senescent phenotype. Thus, DNA damage foci cannot be employed in isolation to detect senescent cells.

\section{SAHF}

In addition to the perceived morphological and biochemical changes, the induction of senescence is also marked by modifications to the chromatin structure $[23,29]$. When $4{ }^{\prime}, 6$-diamidino-2-phenylindole (DAPI) is introduced in certain senescent cells; it elicits a strongly punctate staining pattern of the chromatin structures. These focally dense staining of nuclear domains with DAPI is termed as senescence-associated heterochromatic foci (SAHF). The presence of SAHFs alone do not 


\section{Open Access Journal of Microbiology \& Biotechnology}

necessarily represent senescent cells but is certainly used to mark senescence when accompanied with epigenetic silencing modifications such as trimethylated lysine 9 on histone 3 [30]. Moreover, accumulation of other proteins including HP1, HMGA, histone chaperones HIRA and Asf1, macroH2A, and $\mathrm{pRb}$ at the genetic loci containing proproliferation genes have also been employed for senescence detection [30-33]. Despite their apparent utility for senescence detection, the SAHFs are not universally seen across all senescent cells. The SAHFs are more commonly observed in oncogene induced senescence and closely correlated with increase in p16 INK4a expression, but can also be evoked in cell type and senescence stimuli dependent manner [23]. For these reasons, SAHFs formation alone is rather unreliable marker for senescence detection.

\section{SASP}

Induction of senescence leads to massive alterations in the transcriptional program of several hundreds of proteins. The secretion of such hundreds of factors by senescent cells which include proteases, chemokines, and cytokines, is collectively known as the senescenceassociated secretory phenotype (SASP) $[2,23,29,34,35]$. The SASP exerts a range of effects and at times, the factors involved may have opposing effects depending on the genetic context and cell type. An earlier study reported that persistent DNA damage signaling is required for the secretion of SASP factors. Because DNA damage signaling is not observed in all senescent cells, therefore SASP is not invariably linked to senescence $[4,36]$. Indeed, expression levels of SASP factors are found grossly elevated under non-senescent backgrounds such as inflammation, injury, infection, and prevailing malignances [1]. Currently, no SASP components alone or in combination can unfailingly distinguish senescence from other cellular stresses.

\section{Telomere Shortening}

The telomeric length imposes restriction on the replicative capacity of untransformed normal cells. Abnormally short telomere trigger DDR pathway which in turn blocks the propagation of cells and contribute to the maintenance of chromosomal integrity [37]. It has been proposed that assays measuring telomere erosion and integrity may be useful to identify senescence. A number of approaches are available to examine telomeric length. On account of its precision, the flow cytometry coupled fluorescence in situ hybridization is preferred for visualization and assessment of telomeres. As with other senescence markers, shortened telomere length can be observed in non-senescent settings raising the question on its general applicability for senescence detection. Furthermore, a range of stresses can also induce senescence in human cells in absence of telomeric shortening. For these reasons, senescence determination cannot be solely based on the assessment of length and integrity of telomeres.

\section{Conclusion}

The inability of any of the above markers to unambiguously identify senescence on its own has created problems for faithful detection of senescence. Adding to this problem are the observations that wide range of stimuli can trigger senescence and their mechanism of action are distinct. It is clear that senescence detection will need to be pursued by relying on the combination of senescent markers that are properly adapted to the context under investigation. In this regard, agreeing to the suggestion put forth previously, we feel that stable cell cycle arrest in conjunction with two or more markers, could be acceptable evidences for demonstrating senescence [1]. For example, in an earlier study, we had used SA- $\beta$-gal staining along with p21 induction to mark therapy induced senescence in HNSCC cells. Similarly, combinations of other markers have also been utilized for senescent detection. We feel that availability of a greater pool of senescence markers may enable rapid demonstration of senescence in different contexts. Evolving technologies of genome-wide expression analysis in single-cells and proteomic approaches may be helpful in uncovering novel markers of senescence. For instance, a proteomic study conducted previously had furnished 107 markers of senescence [38]. While such discoveries will undoubtedly improve our understanding of senescence signaling, they may also create new complications arising out of cell heterogeneity and compel us to further refine the definition of senescence.

\section{References}

1. Sharpless NE, Sherr CJ (2015) Forging a signature of in vivo senescence. Nat Rev Cancer 15(7): 397-408.

2. Gadhikar MA, Singh PK (2016) Exploiting Senescence for Cancer Treatment. Mol Bio 5(4).

3. Acosta JC, Gil J (2012) Senescence: a new weapon for cancer therapy. Trends Cell Biol 22(4): 211-219.

4. Kuilman T, Michaloglou C, Mooi WJ, Peeper DS (2010) The essence of senescence. Genes Dev 24(22): 24632479. 


\section{Open Access Journal of Microbiology \& Biotechnology}

5. Dimri GP, Lee X, Basile G, Acosta M, Scott G, et al. (1995) A biomarker that identifies senescent human cells in culture and in aging skin in vivo. Proc Natl Acad Sci USA 92(20): 9363-9367.

6. Itahana K, Campisi J, Dimri GP (2007) Methods to detect biomarkers of cellular senescence: the senescence-associated beta-galactosidase assay. Methods Mol Biol 371: 21-31.

7. Kurz DJ, Decary S, Hong Y, Erusalimsky JD (2000) Senescence-associated (beta)-galactosidase reflects an increase in lysosomal mass during replicative ageing of human endothelial cells. J Cell Sci 113 (Pt 20): 3613-3622.

8. Lee B Y, Han JA, Im JS, Morrone A, Johung K, et al. (2006) Senescence-associated beta-galactosidase is lysosomal beta-galactosidase. Aging Cell 5(2): 187195.

9. Ivanov A, Pawlikowski J, Manoharan I, van Tuyn J, Nelson DM (2013) Lysosome-mediated processing of chromatin in senescence. J Cell Biol 202(1): 129-143.

10. Young AR, Narita M (2010) Connecting autophagy to senescence in pathophysiology. Curr Opin Cell Biol 22(2): 234-240.

11. Bursuker I, Rhodes JM, Goldman R (1982) Betagalactosidase--an indicator of the maturational stage of mouse and human mononuclear phagocytes. J Cell Physiol 112(3): 385-390.

12. Kopp HG, Hooper AT, Shmelkov SV, Rafii S (2007) Beta-galactosidase staining on bone marrow. The osteoclast pitfall. Histol Histopathol 22(9): 971-976.

13. Herbig U, Jobling WA, Chen BP, Chen DJ, Sedivy JM (2004) Telomere shortening triggers senescence of human cells through a pathway involving ATM, p53, and p21(CIP1), but not p16(INK4a). Mol Cell 14(4): 501-513.

14. Rheinwald JG, Hahn WC, Ramsey MR, Wu JY, Guo Z, et al. (2002) A two-stage, p16 (INK4A)- and p53dependent keratinocyte senescence mechanism that limits replicative potential independent of telomere status. Mol Cell Biol 22(14): 5157-5172.

15. Nakao Y, Yang X, Yokoyama M, Ferenczy A, Tang SC, et al. (1997) Induction of p16 during immortalization by HPV 16 and 18 and not during malignant transformation. Br J Cancer 75(10): 1410-1416.
16. Shapiro GI, Edwards CD, Kobzik L, Godleski J, Richards W, et al. (1995) Reciprocal Rb inactivation and p16INK4 expression in primary lung cancers and cell lines. Cancer Res 55(3): 505-509.

17. Abbas T, Dutta A (2009) p21 in cancer: intricate networks and multiple activities. Nat Rev Cancer $9(6): 400-414$.

18. Roninson IB, Broude EV, Chang BD (2001) If not apoptosis, then what? Treatment-induced senescence and mitotic catastrophe in tumor cells. Drug Resist Updat 4(5): 303-313.

19. Gadhikar MA, Sciuto MR, Alves MV, Pickering CR, Osman AA, et al. (2013) Chk1/2 inhibition overcomes the cisplatin resistance of head and neck cancer cells secondary to the loss of functional p53. Mol Cancer Ther 12(9): 1860-1873.

20. Jia L, Li H, Sun Y (2011) Induction of p21-dependent senescence by an NAE inhibitor, MLN4924, as a mechanism of growth suppression. Neoplasia 13(6): 561-569.

21. Mah LJ, El Osta A, Karagiannis TC (2010) gammaH2AX: a sensitive molecular marker of DNA damage and repair. Leukemia 24(4): 679-686.

22. Sharma A, Singh K, Almasan A, (2012) Histone H2AX phosphorylation: a marker for DNA damage. Methods Mol Biol 920: 613-626.

23. Kosar M, Bartkova J, Hubackova S, Hodny Z, Lukas J, et al. (2011) Senescence-associated heterochromatin foci are dispensable for cellular senescence, occur in a cell type- and insult-dependent manner and follow expression of p16(ink4a). Cell Cycle 10(3): 457-468.

24. d'Adda di Fagagna F, Reaper PM, Clay-Farrace L, Fiegler H, Carr P, et al. (2003) A DNA damage checkpoint response in telomere-initiated senescence. Nature 426(6963): 194-198.

25. Takai H, Smogorzewska A, de Lange T (2003) DNA damage foci at dysfunctional telomeres. Curr Biol 13(17): 1549-1556.

26. Bartkova J, Rezaei N, Liontos M, Karakaidos P, Kletsas $D$, et al. (2006) Oncogene-induced senescence is part of the tumorigenesis barrier imposed by DNA damage checkpoints. Nature 444(7119): 633-637. 
27. Di Micco R, Fumagalli M, Cicalese A, Piccinin S, Gasparini P, et al. (2006) Oncogene-induced senescence is a DNA damage response triggered by DNA hyper-replication. Nature 444(7119): 638-642.

28. Frederick MJ, VanMeter AJ, Gadhikar MA, Henderson YC, Yao H, et al. (2011) Phosphoproteomic analysis of signaling pathways in head and neck squamous cell carcinoma patient samples. Am J Pathol 178(2): 548571.

29. Rodier F, Campisi J (2011) Four faces of cellular senescence. J Cell Biol 192(4): 547-556.

30. Narita M, Nũnez S, Heard E, Narita M, Lin AW, et al. (2003) Rb-mediated heterochromatin formation and silencing of E2F target genes during cellular senescence. Cell 113(6): 703-716.

31. Ye X, Brad Z, Rugang Z, Neeta S, Marc L, et al. (2007) Definition of pRB- and p53-dependent and independent steps in HIRA/ASF1a-mediated formation of senescence-associated heterochromatin foci. Mol Cell Biol 27(7): 2452-2465.

32. Narita M, Narita M, Krizhanovsky V, Nuñez S, Chicas A, et al. (2006) A novel role for high-mobility group a proteins in cellular senescence and heterochromatin formation. Cell 126(3): 503-514.
33. Zhang R, Poustovoitov MV, Ye X, Santos HA, Chen W, et al. (2005) Formation of MacroH2A-containing senescence-associated heterochromatin foci and senescence driven by ASF1a and HIRA. Dev Cell 8(1): 19-30.

34. Munoz ED, Serrano M (2014) Cellular senescence: from physiology to pathology. Nat Rev Mol Cell Biol 15(7): 482-496.

35. Salama R, Sadaie M, Hoare M, Narita M (2014) Cellular senescence and its effector programs. Genes Dev 28(2): 99-114.

36. Rodier F, Coppé JP, Patil CK, Hoeijmakers WA, Muñoz DP, et al. (2009) Persistent DNA damage signalling triggers senescence-associated inflammatory cytokine secretion. Nat Cell Biol 11(8): 973-979.

37. Bodnar AG, Ouellette M, Frolkis M, Holt SE, Chiu CP, et al. (1998) Extension of life-span by introduction of telomerase into normal human cells. Science 279(5349): 349-352.

38. Althubiti M, Lezina L, Carrera S, Jukes JR, Giblett SM, et al. (2014) Characterization of novel markers of senescence and their prognostic potential in cancer. Cell Death Dis 5: e1528.

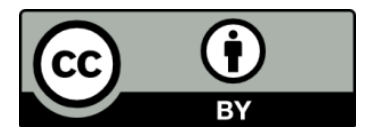


